$5^{\text {th }}$ International Conference on Innovation in Science and Technology

Barcelona - Spain

ISTCONF

7 - 9 December, 2018

\title{
Thermal Performance Analysis of Solar Water Heating System in TRNSYS Under Various Climate Conditions
}

\author{
Naeem Abas ${ }^{1 *}$, Safdar Rasool ${ }^{2}$, Muhammad Irfan ${ }^{3}$, Muhammad Shoaib Saleem ${ }^{4}$ \\ ${ }^{12,3}$ Department of Electrical Engineering, University of Gujrat, Hafiz Hayat Campus, Gujrat \\ ${ }^{4}$ Department of Electrical Engineering, University of Management and Technology Lahore, Sialkot Campus
}

\begin{abstract}
Heating without global warming is a twin challenge faced by global community. Existing synthetic refrigerants have been banned or under a time barred permission under Kyoto Protocol and Paris Agreement. Choice of refrigerant for next generation heating application is major point of interest for industry and policy maker. In this regard, an evacuated glass tube solar water eating system is simulated in TRNSYS software. In the first part, simulation is performed for choice of optimal parameters i.e. size of collector, heat exchanger, tank volume and height. In second stage optimized parameters are used to analyze the performance of system with different natural and synthetic working supercritical fluids. At the end of the study, system performance is evaluated in different cities with different climate by using available weather data in TRNSYS ${ }^{\circledR}$ library and all simulations are performed hourly and then monthly or annual efficiencies and solar fractions are exhibited. The implemented system is designed to fulfill the hot water demand of a single family home with fixed demand of 260 liters/day. It is determined that zero energy utilization roadmap can be traced out with green heating technology without any adverse environmental impacts.
\end{abstract}

Keywords: Heat transfer; Thermal Performance; TRNSYS; Solar Water heating system; super critical fluids 